\title{
Stem Cell in Lingual Epithelia and Papillae
}

\section{Feng Li*}

Shanghai Public Health Clinical Center, Fudan University, Shanghai 201508, China

\begin{abstract}
In mammalian tongue, all the compartments of the lingual epithelium are turned over throughout adult life. There is a continual requirement for proliferation to replace cells lost by differentiation within each compartment. In contrast to remarkably elucidating to the molecular basis for taste perception, too little is known about the mechanism of lingual epithelium and papillae. In this article, we briefly summarize what is known about the different stem cell compartments in lingual epithelium and papillae, and discuss the evidence for existence of stem cell in lingual epithelium and taste bud. We then consider a new model of homeostasis in lingual epithelium and papillae, in which tissue maintenance depends on multiple-stem cell population. Problems revealed by the application of epigenetic marker are also discussed.
\end{abstract}

Keywords: Stem cell; Lingual epithelia; Taste bud; Papillae; Cell genesis

\section{Introduction}

The surface of mammalian tongues consists of a multilayered sheet of keratinocytes, interspersed with filiform (FP) and fungiform (FuP) papillae in the anterior two-thirds, circumvallate $(\mathrm{CV})$ and foliate papillae (FoP) in the posterior one-thirds $[1,2]$. All the compartments of the lingual epithelium are turned over throughout adult life. In the filiform papillae, covering the tongue, proliferation is restricted to cells in the basal cell layer that adhere to an underlying basement membrane. On commitment to terminal differentiation, basal cell lose their attachment to the basement membrane and move into the suprabasal cell layers, then cornified cell layer, ultimately reaching the surface from where they are shed $[3,4]$. Taste bud, included in fungiform, circumvallate and foliate papillae, consist of about 60 or more cells which span the depth of the papilla epithelium, and contain different cell types based on assessment of apical protrusions, organelles and molecular expression. Taste bud cells are believed to be continually turned over about 10 days [5]. Thus, there is a continual requirement for proliferation to replace cells lost by differentiation within each compartment. A key issue in the study of lingual epithelial homeostasis is how these spatially distinct compartments comprising cells of different lineages are maintained.

The field of adult mammalian stem cell biology has been heavily elucidated by lessons learned from several model tissues-hematopoietic stem cells, epithelial stem cells, intestinal stem cells and spermatogonia stem cells [6-8]. Mammalian epidermis consists of the interfollicular epidermis (IFE) with multilayers of keratinocyte, affiliated with hair follicles (HF) and sebaceous glands (SG). In unwounded epidermis, it has always been thought that the own discrete stem cell population contribute to the maintenance of HF, IFE and SG $[9,10]$. In histological views, lingual epithelium is similar to epidermis, classified into stratified squamous epithelium [11]

In this review, we briefly summarize what is known about the different stem cell compartments in lingual epithelium and papillae, and discuss the evidence for existence of stem cell in lingual epithelium and taste bud. We then consider a new model of homeostasis in lingual epithelium and papillae, in which tissue maintenance depends on multiple-stem cell population.

\section{The Plasticity of Lingual Epithelia and Papillae}

\section{Filiform papillae}

Filiform papillae is covered on the dorsal surface of the mammals tongue. Histological, each filiform papillae consist of essentially two parts: a stratified squamous epithelium, including a basal cell layer and a cornified, curved spine projecting from the surface. Each of the filiform papillae has a connective tissue core. There is an interpapillary region between the filiform papillae [3] (Figure 1A).

When slices of 12-14 day fetal tongues, before initial papilla formation, were explanted and grown in organ culture for up to six days, the formed filiform papilla contained both hard and soft keratin. This indicates that the primitive epithelium and mesenchyme contain the necessary information for genesis of filiform papilla and formation of hard and soft keratin [12]. In heterotypic transplants using various combinations of epithelium and mescenchyme from guinea pig ear, trunk, sole of foot, tongue and esophagus, it was revealed that lingual and esophageal epithelium maintained their morphological characteristics when transplanted in combination with mesenchyme from other sources. Epithelia from other sources changed their morphology according to the mesenchyme with which they were transplanted [13]. 30-35 days mutation of TGF- $\beta$ signaling in Keratin14+ cells induce the multiple layers of papilla in the dorsal surface, but lead to the hyperplasia in the ventral surface. Similarly, 40 days mutation of TGF- $\beta$ signaling in NTPDase2+ cells also induce the multiple layers of papilla in the dorsal surface, but did not lead to the hyperplasia in the ventral surface [14]. Those previous data collectively revealed that lingual epithelium pre-store the important information for formation of filiform papilla, and epithelial-mesenchymal interactions play an important role in the regeneration of filiform papilla.

\section{Fungiform papillae}

The adult fungiform papilla is a complex set of tissues and cell types that reside in the specialized multilayered epithelium of the tongue. This unique sensory organ includes: taste buds; a layered and keratinized surface epithelium; and, lateral epithelial walls that extend into the underlying connective tissue to surround a core of lamina propria cells. The core is engorged with blood vessels and bundles of nerve fibers from geniculate and trigeminal sensory ganglia [15] (Figure 1A). More attention has focused on lingual taste buds in the fungiform papillae arranged on the anterior two thirds of the tongue [16], and on the

*Corresponding author: Feng Li, Shanghai Public Health Clinical Center, Fudan University, Shanghai, China, Tel: 8613651824980; E-mail: lifeng30286@shaphc.org

Received May 04, 2018; Accepted May 08, 2018; Published May 15, 2018

Citation: Li F (2018) Stem Cell in Lingual Epithelia and Papillae. Med Chem (Los Angeles) 8: 118-126. doi: 10.4172/2161-0444.1000503

Copyright: $\odot 2018$ Li F. This is an open-access article distributed under the terms of the Creative Commons Attribution License, which permits unrestricted use distribution, and reproduction in any medium, provided the original author and source are credited. 
posterior papillae, the single circumvallate and bilateral foliates (each containing several hundred buds) [5].

Existing data has suggested that if fungiform papillae are not established before filiform papillae during ontogeny, they do not develop at all [17-19]. In another biopsy, the fungiform papillae on one side of the tongue along the midline were removed. Up to 12 months, less papilla with taste buds were found in the operated area, indicating that fungiform papillae had not migrated from areas outside the area of the biopsies, only regenerating in the initial region. When the upper part of the papillae with their taste buds was removed, complete regeneration of papilla was obtained within 14 days. However, when the whole papilla including its base was removed, neither the papilla nor the taste bud regenerated. Taste-buds did not reappear in the regions of the tongue in which fungiform papillae had not regenerated [20]. Other authors transplanted non-gustatory oral epithelia or epidermis into regions innerved by taste nerve fibers, but taste-buds could not be induced in these epithelia even in the presence of nerves that have the capacity to induce them in appropriate epithelia [21]. Another study further shows that the cells of the taste-buds are derived from a unique population of progenitor cells different from that of the surrounding epithelium [22].

In short, based on these previous studies, it seems likely that the important information for regeneration of filiform papilla is pre-stored in the lingual epithelium on the dorsal surface, and that for regeneration of fungiform papilla is pre-stored in the taste bud.

\section{Possible Model of Cell Genesis in Filiform Papillae and Taste Bud}

\section{Filiform papillae}

Early study with $\mathrm{H}^{3} \mathrm{~T}$ has partially revealed the cell-genesis of lingual epithelium. The location of labeled cells was exampled in the epithelium of mouse tongue from a series of animals which were injected with $\mathrm{H}^{3} \mathrm{~T}$ over times ranging from 45 minutes to 7 days. The results show that every cell in the basal layer is labeled within three days, and that all epithelial cells are labeled by five days. It may be suggested that all epithelial cells go through at least one premitotic DNA synthesis in less than five days. The rate of cell labeling is faster on the dorsal surface than the ventral surface. 45 minutes after injection of $\mathrm{H}^{3} \mathrm{~T}$, the labeling incidence of basal cells was found to vary depending on the area of the tongue. The incidence was highest at the tip of the tongue (22\%), intermediate in the mid-section (17\%), and lowest on the back portion (13\%). The turnover times of cells in the basal layer varied depending on the area of the tongue: tip 32 hours, mid 40 hours and back 53 hours. Those cell migration data also indicate two alternate migratory fates for those cells born in the basal cell layer, near the base of the filiform papillae. They may either migrate away from the basement membrane, vertically reach the granular layer with a rapid rate of migration (taking from 3-4 days), or these cells may stay in basement membrane and horizontally migrate slowly through this region (taking from 5-7 days) [3].

Further attempts have been made to clarify the arrangement of the filiform papillae [4]. Hume and Potten further suggest that filiform papillae consist of 4 or 5 columns of differentiating and differentiated cells: anterior column (a), posterior column (p), posterior buttress column (bc), interpapillary regions (ir), dermal papillae (dp). Labeling index (LI) data indicate that LI varies considerably depending on position on the dermal papilla with the highest values tending to be found at the very base of the dermal papilla. They suggest a model revealing probable flow patterns for the cells, and there is presumptive stem cell zone at the base of each dermal papilla for each of the 4 major columns of cells. Hypothetically, filiform papilla is formed by progressive distortion of columnar stacks of epidermal cells (Figure 1A and 1B). They also made an attempt to correlate epithelial thickness, cell production rate and a high degree of organization, but this is not case [23].

\section{Taste bud}

Taste buds are located within specialized papillae on the mammalian tongue. Cells within taste bud are postmitotic [24]. Very small doses of colchicine block the mitotic process in cells at metaphase. The animals were sacrificed at different times after colchicine injection and then histologically examined for normal and degenerating taste buds. Many cells, blocked mitotic divisions, were found in the germinal epithelium of the fungiform papillae as well as around the taste bud over time. Moreover, no mitotic figures were observed inside the taste bud, although most of the taste buds degenerated after 48 hours injection. Radioautography, using tritiated thymidine, reveals that those epithelial cells surrounding the taste bud divide and that some of the daughter cells enter the taste bud and move toward the center. The average life span of a taste bud cell is about 10 days [24].

There has been considerable controversy in the literature concerning the existence of different cell types within verterbrate taste bud (Figure 1B). Farbman [25] observed two cell types, the dark and light cell. Delay et al. [5] morphologically distinguished four different cell types: the basal cell, the dark cell, the intermediate cell, and the light cell. After being injected with ${ }^{3} \mathrm{H}$-thymidine for one hour, the basal cells are labeled within the taste bud. By 12 hours after injection of ${ }^{3} \mathrm{H}$-thymidine, the basal cells are still labeled within the taste bud. Over $90 \%$ of the cells labeled with radioactive tracer in the first 2 days post-injection were basal cells. Those data support the hypothesis that there is only one cell line that undergoes various stages in its lifespan. The differences in morphology of the various cells of the taste bud are due to differences in stages of growth in which they occur at a given time. In contrast, Kinnamon et al. [26] have reported that all cell types except basal cells, such as dark, light, and intermediate cells, in the taste bud have synapses onto sensory nerve fibers. On the other hand, these researchers also observed mitotic cells in taste buds, and further conclude that some taste cells originate from stem cells in the basolateral region of the taste bud [5]. This conclusion differ from that of other researchers [24], who did not report mitotically active cells in taste buds and indicated that taste cells arise exclusively from the surrounding basal epithelial cells. On the other hand, numerous cells were labeled outside the taste bud [5]. In previous study, most of researchers employ the light micro and radioautography. But, Delay employed a high-voltage electron microscopic (HVEM) autoradiography (ARG). Before analyzing the difference, the sensitivity of experimental protocol should be considered.

\section{Labeling Retaining Cells and Activity Cycling Cell in Taste Bud}

Taste buds consist of 50-150 specialized neuroepithelial cells, which are regularly replaced by proliferative cell population. Earlier study shows that taste bud cell arise exclusively from those surrounding epithelial cells $[24,25,27]$. Subsequent study with X chromosomeinactivation mosaic mice strongly supports this argument that taste bud cells arise from local epithelium [22]. Different from this point of view, several labeled basal cells are observed within the taste bud from a mouse perfused 1 or 12 hours after being injected with ${ }^{3} \mathrm{H}$-thymidine, indicating the existence of rapid cycling progenitor cells within that 

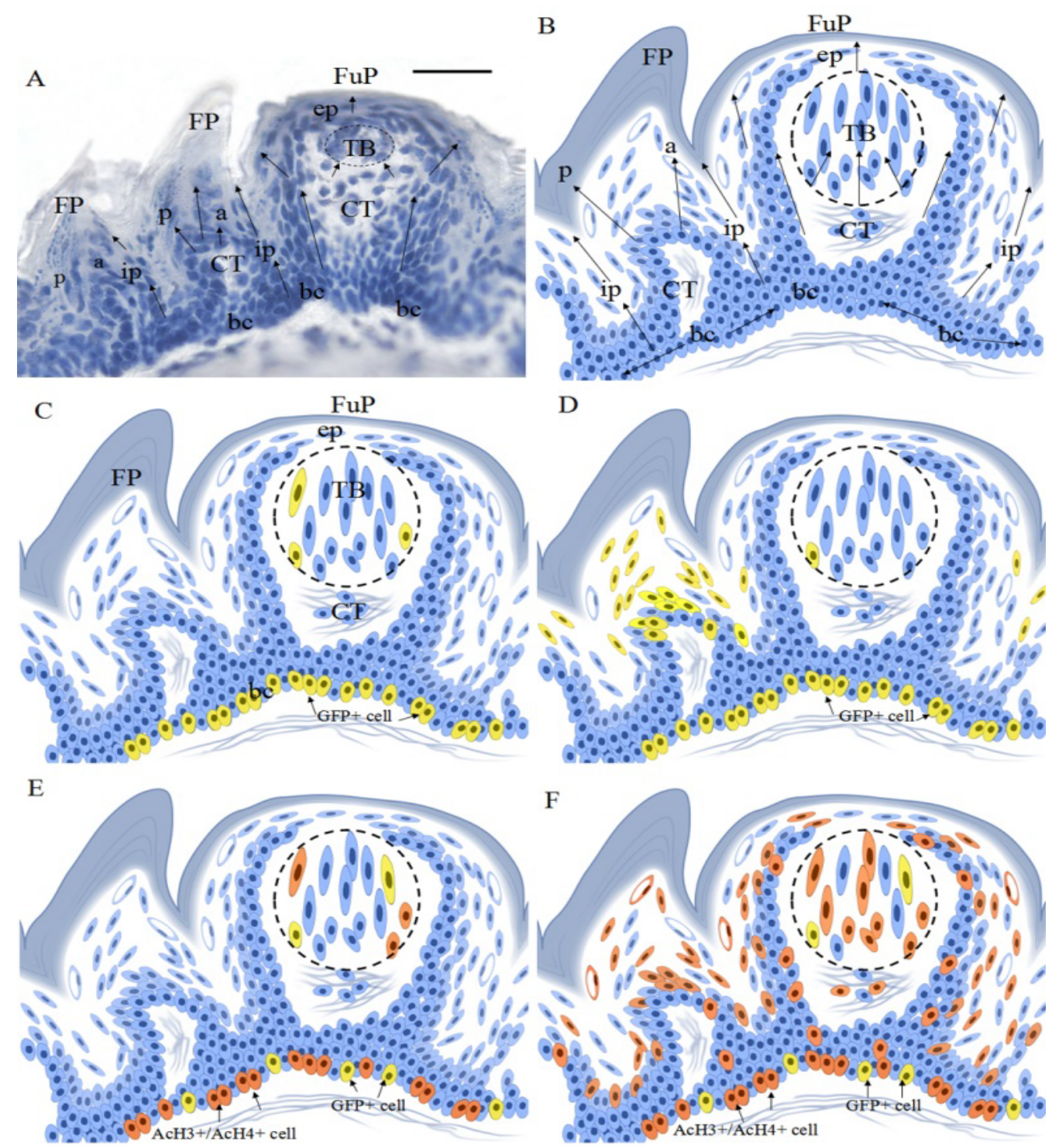

Figure 1: Structure of Murine lingual epithelia and papillae, cell genesis model for filiform and fungiform papillae and Predictions of the cell genesis hypothesis compared with experimental results. (A) Sagittal section of tongue dorsal epithelium including filiform papillae and fungiform papillae. Arrows indicate predicted migration pattern for cells. (B) Filiform papillae is hypothesized to be divided into three compartments: anterior aspect, posterior aspect and interpapillary area. In each compartment, cornified cells are hypothesized to be maintained by the basal cells. In the basal layer, a stem cell generates postmitotic basal cells that subsequently exit the basal layer and migrate vertically to the surface through the suprabasal cell layer. Fungiform papillae include the covering epithelia and taste bud. The basal cells in taste bud are hypothesized to be served as progenitor cells and be able to generate a transient precursor in the taste buds. Other three kinds of cells in taste buds are thought to be derived from those basal cells. The basal cells are hypothesized to be migrated from local epithelium. (C) After 9 or 24 h disruption of TGF- $\beta$ signaling in $\mathrm{K} 14+$ cells, GFP+ cells are observed in basal cell layer of lingual epithelia and papillae. Several GFP+ cells are found within taste bud. (D) After 3D disruption of TGF- $\beta$ signaling in K14+ cells, GFP+ cells are observed in basal cell layer and projection of filiform papillae. In fungiform papillae, GFP+ cells are found in basal cell layer and taste bud. (E) After $5 \mathrm{~h} / 9 \mathrm{~h} / 24 \mathrm{~h}$ disruption of TGF- $\beta$ signaling in $\mathrm{K} 14+$ cells, the $\mathrm{AcH} 3+$ or $\mathrm{AcH} 4+$ cells are predicted to appear in the basal cell layer of lingual epithelia and papillae. Within taste bud, the $\mathrm{AcH} 3+$ or $\mathrm{AcH} 4+$ cells should be observed in basal region. $(\mathrm{F})$ What is observed is that the AcH3+ or AcH4+ cells are distributed through the whole epithelia in lingual epithelium, cutting cross the boundaries of the predicted compartments based on EPU. Arrows indicate potential flow directions for the cells in lingual epithelia and papillae. FP: filiform papillae. FuP: fungiform papillae. TB: taste bud. CT: Connective Tissue. bc: basal cell layer. ep: epithelia layer. ip: interpapillary region. a: anterior. p: posterior. Scale bar $20 \mu \mathrm{m}$.

taste bud [5]. In short, the current experiments have resulted in two different hypotheses, that the stem or/and progenitor cells maintaining adult taste buds are located either inside or outside the taste bud.

In another study, BrdU was used as a thymidine analog to label de novo synthesis of DNA during S- phase of the cell cycle, and BrdU signals were chased from 0.5 to 26 days after the injection. This study shows that the BrdU-positive cells still existed at 26 days, indicating that the lifespan of taste bud cells is heterogeneous, and much longer than previously recognized [28]. In order to figure out taste bud stem or/and progenitor cell populations within or outside taste bud, three different in vivo approaches were used to detect rapid and slow dividing cells related to taste buds. The circadian rhythm in proliferation was considered during all experiments. With two double labeling pulsechase experiments using nucleotide analogues, it is revealed that 
intragemmal cells and perigemmal cells undergo rapid sequential cell divisions, indicating the existence of transient amplifying cells within taste buds. Using the labeling-retaining cell approach, slow-cycling cells were also located in taste bud in doubt [29]. Together, the current results suggest that both taste stem- and progenitor-like cells reside within the taste bud.

\section{Lineage Tracing for Analysis of Stem Cell Fate in Lingual Epithelia and Papillae}

There are three almost insurmountable challenges in studying lingual epithelial stem cell and taste stem cell. The first is that stem cell-specific molecular markers associated with lingual epithelia and papillae are rarely identified so far. Levels of gene expression fluctuate along lingual epithelia and within taste buds; molecular markers may reveal only a subpopulation of stem cell pools. The second is that any in vitro assay to quality and quantitate stem cell will always be imperfect, because it can never completely mimic the vivo environment. Third is that obtaining taste stem cell in vitro is almost impossible so far. Thus, in vivo lineage tracing is a requisite tool for studying stem cell in tongue, for it can reveal the fate of the progeny of an individual stem cell as illustrated by other tissue studies. This methodology allows us to study cellular function in a normal tissue context $[8,30,31]$.

Keratin14 is a stratified epithelial marker and often expressed in mitotically active keratinocytes with keratin 5 . Keratin $14 /$ keratin 5 was reported to be localized in the basal and suprabasal layers of various types of stratified epithelia including the basal cells in taste tissue as well as the surrounding epithelium in the tongue. It is controversial whether Keratin14 is expressed in taste bud. But, several studies reveal the expression of Keratin14 in taste bud, indicating that Keratin14 is not only a molecular marker for keratinocyte in taste tissues [29,32]. Moreover, Keratin 14 is co-expressed in a distinct population of BrdUpositive taste bud cells even after 12 days labeling, indicating the presence of long-lived cells expressing Keratin14 in taste buds. After serial passaging, thymic epithelial cells obtaining from adult thymus were almost positive for K5/K14. These cells can irreversibly follow the fate of hair follicle multipotent stem cells when exposed to an inductive skin microenvironment [33]. The olfactory neuroepithelium undergoes continual neurogenesis. Adult olfactory neuroepithelium neural stem cells were located at the olfactory basal cell (HBCs) layers. Keratin14/ keratin 5 is co-expressed in HBCs, which is a long-lived progenitors that remains quiescent in normal olfactory neuroepithelium. After olfactory neuroepithelium lesioning, cell lineage mapping analysis reveals that Keratin5+ HBCs can regenerate both neuronal and non-neuronal olfactory neuroepithelium lineages [34,35]. Thus, we speculate that Keratin14/keratin5 expression may represent a cell status which has multiple-potential.

With a tamoxifen inducible system (K14-Cre ${ }^{\mathrm{ER}}$-Rosa26-Lac Z), it is shown that K14+ cells give rise to cells within taste buds, as well as to papillary epithelium including fungiform and CV papilla, and lingual epithelium. Provided that keratin14+ cells are located at the basal cell layer of lingual epithelium and taste buds, lac Z lineage labeling patterns reveals the random distribution of K14 lineage cells in lingual epithelium and papillae [36].

Ecto-nucleoside triphosphate diphosphohydrolase 2 (NTPDase2), was found to co-localize with glial glutamate/aspartate transporter (GLAST), which is regarded as a marker of type I cells in taste buds $[37,38]$. NTPDase2, is selectively associated with type- B cells, the stem cells of the subventricular zone (SVZ) [39], and can be associated with GFAP-, nestin-, and DCX positive radial cells of the dentate gyrus
[40], and is also found to be expressed in lingual epithelia. With an inducible genetic cell fating mapping (NTPDase2-rtTA---TetO-Cre--Rosa26-Lac Z or Rosa26-EGFP), NTPDase2+ cells can contribute to regeneration of lingual epithelia and papillae including taste bud. On the other hand, NTPDase2+ cells can also generate the clones in interpapillary epithelia, and form the whole filiform papillae from basal cells to superbasal cells, even spine of filiform papillae. In previous study, spine of filfiorm papillae is always thought to consist of apparent keratohyalin granules $[3,4]$. The current study further reveals the expression of Lac $\mathrm{Z}$ in the hard keratinized spine of filiform papillae. Although NTPDase2 is proved to widely express in lingual epithelia and papillae, Lac Z or GFP signals are observed with lower frequency in lingual epithelia and papilla [41], as revealed in another inducible model---K14-Cre ${ }^{\mathrm{ER}}$-Rosa26-Lac Z. Thus, the current results collectively indicate that NTPDase2+ or K14+ cells contribute to lingual epithelium and papillae in a stochastic manner.

Sonic hedgehog (Shh), only expressed within taste bud [42], plays an important role in papilla development and maintenance. Lineage tracing experiments using adult Shh-ERCre-R26-Tom mice reveals that cells derived from Shh+ cells were located only within taste buds, but not at the surrounding epithelium. Progeny could not be detected in any taste papillae 3 months after induction, indicating that cells marked by shh-expression are transient precursor cell population of adult taste buds, but not a long-term stem cell population [43]. In contrast, Gli1, transcriptional targets of Shh signaling, is widely expressed in lingual epithelium and fungiform papillae including taste bud. With an inducible Gli-CreER-R26-Lac Z mouse, it is revealed that the progeny of Gli1-lineage cells was continually detected in the taste bud, perigemmal cells and basal/superbasal cells of interpapillary epithelium through at least 3 months, indicating that Gli1+ cells belong to a long-term stem cell population in lingual epithelium and papillae. Labeled patterns have similar characteristics to that reported in K14 lineage tracing [36]. It should be noted that Lac $\mathrm{Z}$ signals show a similar labeled pattern from tip tongue to posterior tongue [14]. Taken together, the current results collectively suggest that Shh+ progenitor cells differ from Gli1+ progenitor cells, and Shh+ cells may be shorterterm transient amplifying cells than Gli1+ cells.

Recently, another progenitor cells, Lgr5+ cells, is reported to regenerate taste bud cells and perigemmal cells in circumvallate and foliate papillae of posterior tongue. Lgr5 (leucine-rich repeatcontaining G-protein coupled receptor 5) has been identified to be a molecular marker for adult stem cells in multiple adult tissues including intestine, stomach, and hair follicle [44-46]. With the Lgr5GFP transgenic mice, Lgr5 expression is located at the bottom of the trench area below the CV papilla, near to opening of the ducts of Von Ebner's glands, and those cells at the base of the taste buds below and between the mature taste cells. With the two sets of inducible cell fate mapping system, it is shown that lgr5+ cells in CV tissue are capable of self-renewing, act as long-term stem cells to give rise to multiple cell types both within the taste buds and in the immediately surrounding epithelium. Lgr5+ cells also generate cells in the pore region $[43,47]$. Transaction of the glosspharyngeal nerves bilaterally (GLx) results in degeneration of posterior taste papillae. After four-nine weeks GLX, the progeny of Lgr5 progenitors were present in all taste bud cell types and the surrounding local epithelium, indicating Lgr5+ cells are a progenitor population contributing to taste bud regeneration upon injury [43].

In sum, the current study collectively reveals that NTPDase2+ cells, Keratin 14+ cells and Gli1+ cells are able to generate the clones in lingual epithelium, contribute to the regeneration of filiform papillae, 
and take part in the turnover of taste bud cells. In contrast, Shh+ cells and Lgr5+ cells only contribute to the turnover of taste bud cells. These results also support the hypothesis that multiple populations of biopotential progenitor cells are located at lingual epithelia and within taste buds $[32,36,41]$.

\section{The Characteristics of Basal Cells/Superbasal Cells/ Fishnet Cells}

Lingual epithelium is a specialized epithelia, in which is resided with fungiform papillae and filiform papillae. Most epithelia need to constantly replace damaged or dead cells throughout the life of the animal. Each tissue has its own requisites for homeostasis and regeneration, but the intrinsic properties of stem cells are likely to be similar across tissues.

It has been thought for many years that the epidermis is maintained by two distinct progenitor cell populations stem cell and transit amplifying (TA) cells. The long-lived stem cells can generate their progeny, known as TA cells, which are destined to undergo terminal differentiation. Prior to exiting cell-cycle, TA cells are believed to undergo a limited number of rounds of cell division. In this stem/TA model, the maintenance of the short-lived TA population depends on the continual slow cycling of stem cell population [48,49].

By far the most influential model of the role of stem and TA cells in epidermal homeostasis is that of the epidermal proliferative unit (EPU). Stem/TA cells divide and vertically migrate into superbasal layer when they leave the basal layer. Each stack of cornified layer cells will be descended from the cells in the basal layer that locate directly beneath it, forming an EPU [50]. However, more and more results do not always support the classic concept of the EPU [10]. Cells in the suprabasal layers differentiate, and can migrate laterally as well as vertically. Those studies challenge the EPU hypothesis that cornified layer cells are derived from the basal cells immediately beneath them [51-53].

According to morphological classification, the interpapillary regions and the ventral surface of the tongue are classified into stratified soft-cornified epithelia, and filiform paillae is stratified hard-cornified epithelia. Reference to this EPU model, Hume and Potten [4] in detail described the ordered columnar structure of mouse filiform papillae and possible cell movement flow. In the stem/TA model (EPU), basal cells divide and proliferate in basal cell layer, move into suprabasal cell layer, then undergo terminal differentiation and migrate into the granular layer. Finally, those cells migrate into the most superficial layer-cornified cell layer (Figure 1A and 1B).

As a matter of fact, lingual epithelium shares a common pathological change with epidermis after mutation of several important signaling pathway in Keratin14+ or 5+ cells. Conditional expression of the epidermal growth factor receptor---ErbB2 in K14+ cells results in reversible hyperplasia in stratified epithelia including skin, corna, tongue and esophagus. Increase of epithelial thickness was observed in both dorsal and ventral surface of the tongue [54]. Conditional mutation of the transforming growth factor- $\beta$ (TGF- $\beta$ ) signaling pathway in K14+ cells also caused hyperplasia on the ventral surface of the tongue, and hyper-proliferation of filiform papillae on the dorsal surface of the tongue. After a long-term mutation of TGF signaling pathway, the condition of the mice had deteriorated to a severe degree with necrotizing ears and bleeding skin [14]. Genetic loss of Hox13 leads to fractured hair at the skin surface, and malformed nails with a flatter appearance. The spine of filiform papillae also appeared to have broken off on the dorsal surface of tongue from Hox13 homozygotes
[55]. The p63 gene, a homologue of the tumor-suppressor p53, is strongly expressed in the basal, or progenitor cells of several epithelial structures. Genetic deletion of p63 lead to the absence of a stratified epidermis, which lack the characteristic structure of basal, suprabasal and cornified layers, and associated hair follicles. Histological analysis of tongue from p63 mutants reveals an absence of the normally stratified epithelium on the tongue, and its replacement by one or two layers of cuboidal epithelium [56]. In short, those results indicate that lingual epithelium appear to share a similar development mechanism with other stratified epithelium.

Filifiorm papillae, affiliated to lingual epithelium, have been showed to be a unique structure [3,4,32]. More and more study provides evidences that there existed specific development mechanism in filiform papillae. In the adult mouse, Pax9 is expressed in the tongue, esophagus, salivary glands, and thymus, but not in skin [57]. In the absence of Pax9, filiform papillae lack an anterior-posterior polarity as well as a cornified layer. Several hard keratins (K1-5, K1-24 and K2-16) are also lost from lingual epithelium in the absence of Pax9 [58]. Over-expression of Sox2 in $\mathrm{K} 5+$ cells completely eliminates well-differentiated filiform papillae expressing trichohyalin, AE15. In contrast, many small, papilla-like domes were observed in the lingual epithelium [59]. Shh signaling play a important role in fungiform induction, development and patterning $[16,60]$. Effectors of Shh signaling in vertebrates are Gli proteins--Gli1, Gli2, Gli3 [61]. Conditional over-expression of Gli2 transcription factor in $\mathrm{K} 5+$ cells completely alters the surface architecture of tongue. Scanning electron micrographs (SEM) shows that fungiform papillae do not have an obvious taste pore, and the sharp, spinous extensions have eliminated from filiform papillae [62]. Over-expression of Noggin (BMP antagonist) in $\mathrm{K} 14+$ cells changes spine shape of the filiform paillae at the anterior part and edge of the tongue from usual rounded types to abnormally pointed tip. Spines of the filiform papilla in K14Noggin mice are not found to significantly differ from that of control mice in the intermolar eminence. This indicates that development of filiform papillae may be regulated by different molecular, dependent on location of tongue [63]. In short, the current study collectively show that conditional disruption of signaling pathway in basal cell layer (K14+ or K5+ cells) can lead to abnormal development of filiform papillae, even loss of spines. This may always be explained by abnormal proliferation of basal cells. This is not case.

Recently, our study with conditional disruption of TGF- $\beta$ signaling pathway in K14+ or NTPDase2+ cells allow us to reconsider the development mechanism of lingual epithelium and filiform papillae. A large number of studies in hair follicle stem cells $[64,65]$ and olfactory stem cell $[34,35]$ reveal that cycling tissue likely harbors a second quiescent stem cell population that becomes active only on injury. After mutation of TGF- $\beta$ signaling in K14+ and NTPDase $2+$ cells or DTA expression in NTPDase2+ cells, cells with the higher AcH3 and AcH4 expression rapidly increased in all layers including basal, suprabasal, conified cell layers. However, dividing cells are only observed in basal cell layer. Thus, it is impossible that cells with the higher $\mathrm{AcH} 3$ and AcH4 level in suprabasal/cornified layer migrates from those cells in basal layer by laterally as well as vertically. Does quiescent stem cell population become active in the current experiment condition? Or normally quiescent differentiated cells can act as progenitor cells after injury-"facultative stem cell"? The current studies have suggested "neutral drift" hypothesis during the proliferation and differentiation of stem cells. Loss of stem cell is compensated by proliferation of neighbors following an active and stochastic pattern of behavior, resulting in progressive expansion of some clones and extinction of others $[18,66]$. 


\section{Interplay between Signal Transduction Pathway and Epigenetic Regulation may Contribute to the Abnormal Development of Filiform Papillae and Proliferation of Lingual Epithelia}

TGF- $\beta$, which signals through activation of TGF- $\beta$ receptors type I and Type II and phosphorylation of the signaling mediators Smad 2 and Smad3, induce multiple cellular responses on various cell types $[67,68]$. Conditional genetic loss of TGF- $\beta$ RII reveals the tumor suppressor characteristics of TGF- $\beta$ signaling pathway in several epithelial tissues $[69,70]$. However, epidermal or hair follicle abnormalities are not observed in those transgenic mice expressing a dominant-negative form of TGF- $\beta$ RII [71] or conditional genetic deletion of TGF- $\beta$ RII in $\mathrm{K} 14+$ cells [70] or in K5+ cells [72]. It seems that TGF- $\beta$ signaling pathway regulate stem/progenitor cell proliferation in a contextdependent manner. Genetic loss of TGF- $\beta$ RII shortens cell cycle, cause cell's withdrawal from cell cycle without affecting cell-fate decisions and progenitor-cell proliferation [73].

The double-transgenic K14-rtTA-PTR or NTPDase2-rtTA-PTR mice co-express EGFP and the dominant-negative Tgfbr2 genes upon treatment with Doxycycline (Dox) in K14+ [32] or NTPDase2+ [41] lingual epithelial cells. Mutation of TGF- $\beta$ signaling may lead to K14+ or NTPDase2+ progenitor cell exiting from cell cycle without affecting cell-fate decisions and transient amplifying cell proliferation. Thus, two models allow us to compare cell fate between K14+ or NTPDase2+ progenitor cells by tracing GFP expression. After mutation of TGF- $\beta$ signaling, the fate of the K14+ cells was to develop into keratinocytes, and NTPDase + cells became transit amplifying cells (TACs), a fate different from that of K14+ cells $[14,32,41,74]$.

Conditional mutation of TGF- $\beta$ signaling pathway in K14+ cells or NTPDase2+ cells results in the thicken epithelium and multiple developing papillae on the dorsal surface. However, it is only in $\mathrm{K} 14+$ cells that conditional mutation of TGF- $\beta$ signaling pathway lead to lingual epithelial hyperplasia on the ventral surface during the 40 days Dox induction. It seems that TGF- $\beta$ signaling pathway regulating progenitor cells proliferation might be tongue area-specific and affected by cellular context. Many classical tissue recombination experiments support the notion that epithelial appendage and tissue formation is instructed by mesenchymal signals, and inductive ability of mesenchymal tissues regulate epithelial cell-fate determination $[75,76]$. The formation of multiple developing papillae on the dorsal surface in two mutation models also suggests that the differentiation of mesenchymal cells into a proper organizing center may be in the control of a second signal originating from the epithelial cells. Taken together, the fate induction of epithelial cells may be the reciprocal inductive interactions between the mesenchyme and the overlying epithelium. Furthermore, the balance between proliferation and differentiation appears to follow from intrinsic (cell-autonomous) regulation $[8,14,30,32,41]$.

The current results showed a different acetylation pattern of histone $\mathrm{H} 3$ and histone $\mathrm{H} 4$ between the two mutant mouse models. In CV papillae, it was easy to distinguish a difference between the two models. In NTPDase2-rtTA-PTR mice, the higher levels of acetylated histone $\mathrm{H} 3$ and histone $\mathrm{H} 4$ were found in both taste buds and epithelia of CV papillae, while they were found only in taste buds of K14-rtTA-PTR mice. This observation further indicated that K14+ cells represented a specialized progenitor which only occupied a specific position, while NTPDase2+ cells were more widely distributed in lingual epithelia (Supplementary Figure 1A-1J).
On the other hand, the higher acetylation of histone $\mathrm{H} 3$ and histone $\mathrm{H} 4$ represented the progression of tongue epithelial stem cells through different stages of development and differentiation. After $5 \mathrm{~h}$ of Dox induction, we only found weak GFP expression in the taste buds of the K14-rtTA-PTR model. However, GFP+ cells were observed within the taste buds at $24 \mathrm{~h} \mathrm{[32]} \mathrm{and} \mathrm{in} \mathrm{basal} \mathrm{cell} \mathrm{layer} \mathrm{of} \mathrm{lingual} \mathrm{epithelia} \mathrm{at}$ the tip of the tongue at $9 \mathrm{~h}$ (Figure 1C and 1D; Supplementary Figure $2 \mathrm{~A}-2 \mathrm{I}$ ), indicating that less than $24 \mathrm{~h}$ in $\mathrm{TB}$ and less than $9 \mathrm{~h}$ in the tip of tongue was needed for the progenitors to become mature cells after disruption of TGF- $\beta$ signaling in K14+ lineage cells [32]. Theoretically, we should observe AcH3+/AcH4+ cells in basal cell layers in lingual epithelia and taste bud (Figure 1E). However, in the K14-rtTA-PTR model, we indeed found cells with higher levels of acetylated histone $\mathrm{H} 3$ and histone H4 in taste bud at $9 \mathrm{~h}$ (Figure 1F; Supplementary Figure $1 \mathrm{C}$ and $1 \mathrm{D}$ ) and the tip of the tongue at $5 \mathrm{~h}$ and $9 \mathrm{~h}$ (Figure 1F; Supplementary Figure 3E-3L), which should not have originated from K14+ cells at that time but rather were cells in an earlier stage of maturation. In addition, cells with higher levels of acetylated histone $\mathrm{H} 3$ and histone $\mathrm{H} 4$ were widely distributed in lingual epithelia with (Supplementary Figure 4E, 4I, 4G and 4K) or without (Supplementary $4 \mathrm{~F}, 4 \mathrm{~J}, 4 \mathrm{H}$ and $4 \mathrm{~L}$ ) filiform papillae of posterior tongue at 5 or $9 \mathrm{~h}$ Dox induction. To keep the normal balance of the cell migration stream, those immature cells located downstream should have been removed when TGF- $\beta$ signaling was interrupted in both mouse models and disturbed the maturation process. However, those immature cells failed to complete modifications such as deacetylation in the final maturation stage due to a shortened cell cycle.

After analysis of the higher $\mathrm{AcH} / \mathrm{AcH} 4$ expression between mutation of TGF- $\beta$ signaling and DTA expression in NTPDase2+ cells, different expression pattern is observed in intragemmal and perigemmal cells of CV papillae. The taste bud cells with the higher $\mathrm{AcH} 3 / \mathrm{AcH} 4$ expression were observed in two models. However, the perigemmal cells with the higher $\mathrm{AcH} 3 / \mathrm{AcH} 4$ expression were only detected after mutation of TGF- $\beta$ signaling. It further means that a different cell genesis may be occurred in lingual epithelia and taste bud of CV papillae (Supplementary Figure 2E-2J).

Another finding in our study is the wide distribution of cells with higher levels of acetylated histone $\mathrm{H} 3$ and histone $\mathrm{H} 4$ across the whole lingual epithelia both in tip-tongue (Supplementary Figure 3M-3T) and posterior-tongue (Supplementary Figure $4 \mathrm{M}-4 \mathrm{P}$ ) after TGF- $\beta$ signal disruption or DTA expression in NTPDase2+ cells. Molecular mechanism leading to pathological changes is distinguished between two models. The frontier is the disruption of TGF- $\beta$ signal pathway in NTPDase2+ cells, and then the latter is the inhibition of protein synthesis caused by the DTA expression in NTPDase2+ cells. However, the reason why $\mathrm{AcH} 3+/ \mathrm{AcH} 4+$ cells are rapidly increased in the whole epithelia remains unclear. Anyway, the current study reveals that NTPDase2+ cells play a critical role in maintaining the normal microenvironment of lingual epithelia, and any damage to NTPDase2+ cells function will increase the $\mathrm{AcH} 3+/ \mathrm{AH} 4+$ cells in lingual epithelia.

The next question to answer was why the higher acetylated histone $\mathrm{H} 3$ and histone $\mathrm{H} 4$ were distributed throughout the whole tongue epithelia after Dox induction in both models, and not limited to the basal layer. In essence, our results were inconsistent with the classic EPU model $[77,78]$, which states that stem cells divide in the basal layer, then move progressively up to the suprabasal cell layer, the granular layer and cornified layer (Figure $1 \mathrm{~A}$ and $1 \mathrm{~B}$ ). However, according to the model of cell migration stream in the adult tongue, NTPDase2+ stem cells are located in the filiform papillae, fungiform papillae, CV papillae and epithelia. Cell genesis occurring within the taste bud is different 
from that of the filiform papillae and epithelia. K14+ stem cells are located further down in the cell migration stream than the NTPDase2+ facultative stem cells, which play a very important role in supporting the normal migration. In our study, we observed that disruption of TGF- $\beta$ signaling even for a short time functionally damaged the NTPDase2+ facultative stem cells, which caused a disturbance and pushed the immature cells out from all along the cell migration stream. By contrast, the disrupted TGF- $\beta$ signaling only caused the downstream proliferating K14+ cells to leave the cell migration stream. Thus, different patterns of the higher AcH4+ expression were observed between the two models.

Other studies have reported observations of epithelial stem cell migration failing to conform to predicted EPU boundaries and challenge the model describing the epidermis as being organized into discrete EPUs [77,78]. Studies of chimeric mice expressing different genetic markers revealed large lines of marker expression in the epidermis, the boundaries of which transverse the EPUs [79]. Later studies showed the persistence of columns of labeled cells in mouse IFE that are larger and more irregularly shaped than the classic EPU $[51,52,78,80,81]$. These studies also revealed that epithelial cells can differentiate laterally as well as vertically [51-53].

Taken together, all these observations suggested that mature cells are not progressively generated from the basal layer in adult tongue epithelia. In addition, Wnt [30,82], Notch [83] and Bone Morphogenetic Protein (BMP) pathways [84] have been reported to involve in the regulation of epithelial stem cell proliferation. The components of the TGF- $\beta$ [85], Notch $[86]$ and $\beta$-Catenin/Wnt $[87,88]$ signaling pathways are expressed in lingual epithelia and/or taste buds. Further histological analysis indicates that interplay between signal transduction pathway and epigenetic modification may contribute to the stem cell development in lingual epithelia and papillae [14,74].

\section{Challenging the Status Quo}

The earlier study has shown the varied turnover rate of lingual epithelial cell, dependent on area of tongue. The current results further reveal that K14 expression varies dependent on the location of tongue and papillae. In addition, $\mathrm{AcH} 3$ and $\mathrm{AcH} 4$ expression also show the variation along the lingual epithelia from tip to posterior tongue. In our limited survey, conditional mutation of several signaling pathway in K14+ or K5+ cells, such as Shh [62], TGF- $\beta$ [14], BMP [63], lead to the shape change of spine of filiform papillae and the different pathological change, dependent on the area of tongue. All these results provide an intriguing issue that lingual epithelia and appendage-filiform papillae may have a unique development mechanism.

Effort to address the factors controlling the development of lingual epithelia and papillae have placed focus on mechanism resulting in stem cell longevity and clonal persistence in adult lingual tissue with inducible genetic labeling systems. However, complexity of microenvironment confuses our understanding on the molecular mechanism leading to balanced stochastic fate in lingual epithelium. Cycling tissues including intestinal epithelium, epidermal epithelium, corneal epithelium and lingual epithelium share common characteristics in balancing between proliferation and differentiation, but it seems that more variation may be observed in lingual epithelium. There are four types of papillae in lingual epithelium including filiform, fungiform, foliate and CV papillae. So far, the molecular mechanisms regulating lingual papillae development remain unclear. What factors control the balance between proliferation and differentiation in lingual epithelia and filiform papillae? What factors lead to dysregulation between proliferation and differentiation in lingual epithelia and filiform papillae during disease and cancer?

\section{Conclusion}

In this review, we have limited our focus to comparative features of stem cell in lingual epithelium and papillae, and what we might gain from such comparisons. It will be interesting to ascertain what features lingual epithelium will share with other stratified epithelium including corneal and skin epithelia. Many concepts, such as quiescence stem cell, TA cells, symmetric-asymmetric divisions, and neural drift, are by no means limited to epidermis. Whether adult lingual epithelia stem cells conform to similar patterns of stochastic fate remains an important open question.

\section{Acknowledgments}

This work is supported by the Start-on Funding from Shanghai Public Health Clinical Center (KY-GW-2017-06).

\section{References}

1. Breslin PAS, Huang L (2006) Human taste: peripheral anatomy, taste transduction, and coding. Adv Otorhinolaryngol 63: 152-190.

2. Breslin PA, Spector AC (2008) Mammalian taste perception. Current Biology 18: R148-R155.

3. Cameron IL (1966) Cell Proliferation, migration, and specialization in the epithelium of the mouse tongue. J Exp Zool 163: 271-283.

4. Hume WJ, Potten CS (1976) The ordered columnar structure of mouse filiform papillae. J Cell Sci 22: 149-160.

5. Delay RJ, Kinnamon JC, Roper SD (1986) Ultrastructure of mouse vallate taste buds: II. Cell types and cell lineage. The Journal of Comparative Neurology 253: $242-252$.

6. Klein AM, Nakagawa T, Ichikawa R, Yoshida S, Simons BD (2010) Mouse germ line stem cells undergo rapid and stochastic turnover. Cell Stem Cell 7: 214-224.

7. Simons BD, Clevers H (2011) Stem cell self-renewal in intestinal crypt. Exp Cell Res 317: 2719-2724.

8. Simons BD, Clevers $H(2011)$ Strategies for homeostatic stem cell self-renewal in adult tissues. Cell 145: 851-862.

9. Clayton E, Doupe DP, Klein AM, Winton DJ, Simons BD, et al. (2007) A single type of progenitor cell maintains normal epidermis. Nature 446: 185-189.

10. Jones PH, Simons BD, Watt FM (2007) Sic transit gloria: farewell to the epidermal transit amplifying cell? Cell Stem Cell 1: 371-381.

11. Iwasaki $S$ (2002) Evolution of the structure and function of the vertebrate tongue. J Anat 201: 1-13.

12. Farbman Al (1973) Differentiation of lingual filiform papillae of the rat in organ culture. Arch Oral Biol 18: 197-202.

13. Billingham RE, Silvers WK (1968) The use of tolerance to reduce the complexity of reactions to antigen mixtures. Cancer Res 28: 1404-1409.

14. Li F, Zhou M (2013) Conditional expression of the dominant-negative TGFbeta receptor type II elicits lingual epithelial hyperplasia in transgenic mice. Developmental Dynamics 242: 444-455.

15. Mistretta CM, Baum BJ (1984) Quantitative study of taste buds in fungiform and circumvallate papillae of young and aged rats. J Anat 138: 323-332.

16. Mistretta CM, Liu HX (2006) Development of fungiform papillae: patterned lingual gustatory organs. Arch Histol Cytol 69: 199-208.

17. Farbman $\mathrm{Al}$ (1971) Differentiation of foetal rat tongue homografts in the anterior chamber of the eye. Arch Oral Biol 16: 51-57.

18. Farbman $\mathrm{Al}$ (1971) Differentiation of oral epithelium and taste bud development J Dent Res 50: 1422-1427. 
19. Mackenzie IC, Hill MW (1981) Maintenance of regionally specific patterns of cell proliferation and differentiation in transplanted skin and oral mucosa. Cell Tissue Res 219: 597-607.

20. Hellekant G, Kasahara Y, Farbman Al, Harada S, Segerstad CH (1987) Regeneration ability of fungiform papillae and taste-buds in rats. Chem Senses 12: 459-465.

21. Zalewski AA (1972) Regeneration of taste buds after transplantation of tongue and ganglia grafts to the anterior chamber of the eye. Exp Neurol 35: 519-528.

22. Stone LM, Finger TE, Tam PP, Tan SS (1995) Taste receptor cells arise from local epithelium, not neurogenic ectoderm. Proc Natl Acad Sci USA 92: 19161920.

23. Potten CS (1975) Epidermal cell production rates. The Journal of Investigative Dermatology 65: 488-500.

24. Beidler LM, Smallman RL (1965) Renewal of cells within taste buds. J Cell Biol 27: 263-272.

25. Farbman Al (1980) Renewal of taste bud cells in rat circumvallate papillae. Cell Tissue Kinet 13: 349-357

26. Kinnamon JC, Taylor BJ, Delay RJ, Roper SD (1985) Ultrastructure of mouse vallate taste buds. I. Taste cells and their associated synapses. The Journal of Comparative Neurology 235: 48-60.

27. Murray RG, Murray A (1971) Relations and possible significance of taste bud cells. Contrib Sens Physiol 5: 47-95

28. Hamamichi R, Asano-Miyoshi M, Emori Y (2006) Taste bud contains both short-lived and long-lived cell populations. Neuroscience 141: 2129-2138.

29. Sullivan JM, Borecki AA, Oleskevich S (2010) Stem and progenitor cell compartments within adult mouse taste buds. The European Journal of Neuroscience 31: 1549-1560

30. Blanpain C, Horsley V, Fuchs E (2007) Epithelial stem cells: turning over new leaves. Cell 128: 445-458.

31. Fuchs E, Horsley V (2011) Ferreting out stem cells from their niches. Nat Cell Biol 13: 513-518

32. Li F, Zhou M (2012) Local microenvironment provides important cues for cell differentiation in lingual epithelia. PLoS One 7: e35362.

33. Bonfanti P, Claudinot S, Amici AW, Farley A, Blackburn CC, et al. (2010) Microenvironmental reprogramming of thymic epithelial cells to skin multipotent stem cells. Nature 466: 978-982.

34. Iwai N, Zhou Z, Roop DR, Behringer RR (2008) Horizontal basal cells are multipotent progenitors in normal and injured adult olfactory epithelium. Stem Cells 26: 1298-1306.

35. Leung CT, Coulombe PA, Reed RR (2007) Contribution of olfactory neura stem cells to tissue maintenance and regeneration. Nat Neurosci 10: 720-726.

36. Okubo T, Clark C, Hogan BL (2009) Cell lineage mapping of taste bud cells and keratinocytes in the mouse tongue and soft palate. Stem Cells 27: 442-450.

37. Bartel DL, Sullivan SL, Lavoie EG, Sevigny J, Finger TE (2006) Nucleoside triphosphate diphosphohydrolase-2 is the ecto-ATPase of type I cells in taste buds. J Comp Neurol 497: 1-12.

38. Lawton DM, Furness DN, Lindemann B, Hackney CM (2000) Localization of the glutamate-aspartate transporter, GLAST, in rat taste buds. The European Journal of Neuroscience 12: 3163-3171

39. Braun N, Sevigny J, Mishra SK, Robson SC, Barth SW, et al. (2003) Expression of the ecto-ATPase NTPDase2 in the germinal zones of the developing and adult rat brain. Eur J Neurosci 17: 1355-1364.

40. Shukla V, Zimmermann H, Wang L, Kettenmann H, Raab S, et al. (2005) Functional expression of the ecto-ATPase NTPDase2 and of nucleotide receptors by neuronal progenitor cells in the adult murine hippocampus. $J$ Neurosci Res 80: 600-610.

41. Li F, Cao J, Zhou M (2012) NTPDase2+ Cells Generate Lingual Epithelia and Papillae. Frontiers in Genetics 3: 255

42. Miura H, Barlow LA (2010) Taste bud regeneration and the search for taste progenitor cells. Arch Ital Biol 148: 107-118.

43. Takeda N, Jain R, Li D, Li L, Lu MM, et al. (2013) Lgr5 Identifies Progenito Cells Capable of Taste Bud Regeneration after Injury. PLoS One 8: e66314.

44. Barker N, Huch M, Kujala P, van de Wetering M, Snippert HJ, et al. (2010) Lgr5(+ve) stem cells drive self-renewal in the stomach and build long-lived gastric units in vitro. Cell Stem Cell 6: 25-36.

45. Barker N, van Es JH, Kuipers J, Kujala P, van den Born M, et al. (2007) Identification of stem cells in small intestine and colon by marker gene Lgr5. Nature 449: 1003-1007.

46. Jaks V, Barker N, Kasper M, van Es JH, Snippert HJ, et al. (2008) Lgr5 marks cycling, yet long-lived, hair follicle stem cells. Nat Genet 40: 1291-1299.

47. Yee KK, Li Y, Redding KM, Iwatsuki K, Margolskee RF, et al. (2013) Lgr5EGFP marks taste bud stem/progenitor cells in posterior tongue. Stem Cells 31: 992-1000.

48. Allen TD, Potten CS (1974) Fine-structural identification and organization of the epidermal proliferative unit. J Cell Sci 15: 291-319.

49. Potten CS (1981) Cell replacement in epidermis (keratopoiesis) via discrete units of proliferation. Int Rev Cytol 69: 271-318.

50. Mackenzie IC (1970) Relationship between mitosis and the ordered structure of the stratum corneum in mouse epidermis. Nature 226: 653-655.

51. Ito M, Liu Y, Yang Z, Nguyen J, Liang F, et al. (2005) Stem cells in the hair follicle bulge contribute to wound repair but not to homeostasis of the epidermis Nat Med 11: 1351-1354.

52. Ro S, Rannala B (2004) A stop-EGFP transgenic mouse to detect clonal cell lineages generated by mutation. EMBO Rep 5: 914-920.

53. Ro S, Rannala B (2005) Evidence from the stop-EGFP mouse supports a nichesharing model of epidermal proliferative units. Exp Dermatol 14: 838-843.

54. Xie W, Chow LT, Paterson AJ, Chin E, Kudlow JE (1999) Conditional expression of the ErbB2 oncogene elicits reversible hyperplasia in stratified epithelia and up-regulation of TGFalpha expression in transgenic mice. Oncogene 18: 3593 3607.

55. Godwin AR, Capecchi MR (1998) Hoxc13 mutant mice lack external hair Genes Dev 12: 11-20.

56. Yang A, Schweitzer R, Sun D, Kaghad M, Walker N, et al. (1999) p63 is essential for regenerative proliferation in limb, craniofacial and epithelial development. Nature 398: 714-718.

57. Peters H, Schuster G, Neubuser A, Richter T, Hofler H, et al. (1997) Isolation of the Pax9 cDNA from adult human esophagus. Mammalian genome 8: $62-64$

58. Jonker L, Kist R, Aw A, Wappler I, Peters H (2004) Pax9 is required for filiform papilla development and suppresses skin-specific differentiation of the mammalian tongue epithelium. Mech Dev 121: 1313-1322.

59. Okubo T, Pevny LH, Hogan BLM (2006) Sox2 is required for development of taste bud sensory cells. Genes Dev 20: 2654-2659.

60. Mistretta CM, Liu HX, Gaffield W, MacCallum DK (2003) Cyclopamine and jervine in embryonic rat tongue cultures demonstrate a role for Shh signaling in taste papilla development and patterning: fungiform papillae double in number and form in novel locations in dorsal lingual epithelium. Dev Biol 254: 1-18.

61. Saha K, Schaffer DV (2006) Signal dynamics in Sonic hedgehog tissue patterning. Development 133: 889-900.

62. Liu HX, Ermilov A, Grachtchouk M, Li L, Gumucio DL, et al. (2013) Multiple Shh signaling centers participate in fungiform papilla and taste bud formation and maintenance. Dev. Biol. 382: 82-97.

63. Kawasaki K, Porntaveetus T, Oommen S, Ghafoor S, Kawasaki M, et al. (2011) Bmp signalling in filiform tongue papillae development. Arch Oral Biol 57: 805813

64. Jensen KB, Collins CA, Nascimento E, Tan DW, Frye M, et al. (2009) Lrig1 expression defines a distinct multipotent stem cell population in mammalian epidermis. Cell Stem Cell 4: 427-439.

65. Snippert HJ, van der Flier LG, Sato T, van Es JH, van den Born M, et al 
(2010) Intestinal crypt homeostasis results from neutral competition between symmetrically dividing Lgr5 stem cells. Cell 143: 134-144.

66. Klein AM, Simons BD (2011) Universal patterns of stem cell fate in cycling adult tissues. Development 138: 3103-3111.

67. Bierie B, Moses HL (2006) TGF-beta and cancer. Cytokine Growth Factor Rev 17: $29-40$.

68. Siegel PM, Massague J (2003) Cytostatic and apoptotic actions of TGF-beta in homeostasis and cancer. Nat Rev Cancer 3: 807-821.

69. Biswas S, Chytil A, Washington K, Romero-Gallo J, Gorska AE, et al. (2004) Transforming growth factor beta receptor type II inactivation promotes the establishment and progression of colon cancer. Cancer Res 64: 4687-4692.

70. Guasch G, Schober M, Pasolli HA, Conn EB, Polak L, et al. (2007) Loss of TGF beta signaling destabilizes homeostasis and promotes squamous cell carcinomas in stratified epithelia. Cancer Cell 12: 313-327.

71. Amendt C, Schirmacher $P$, Weber H, Blessing M (1998) Expression of a dominant negative type II TGF- $\beta$ receptor in mouse skin results in an increase in carcinoma incidence and an acceleration of carcinoma development. Oncogene 17: 25-34.

72. Lu SL, Herrington H, Reh D, Weber S, Bornstein S, et al. (2006) Loss of transforming growth factor-beta type II receptor promotes metastatic head-andneck squamous cell carcinoma. Genes Dev 20: 1331-1342.

73. Falk S, Wurdak H, Ittner L, Ille F, Sumara G, et al. (2008) Brain Area-Specific Effect of TGF- $\beta$ S Signaling on Wnt-Dependent Neural Stem Cell Expansion. Cell Stem Cell 2: 472-483.

74. Li F, Zhou M (2014) The interactions of TGF-beta signalling pathway and Jagged2/Notch1 pathway induce acanthosis in lingual epithelia. Pathology (Phila) 46: 555-565.

75. Ferraris C, Chevalier G, Favier B, Jahoda CA, Dhouailly D (2000) Adult corneal epithelium basal cells possess the capacity to activate epidermal, pilosebaceous and sweat gland genetic programs in response to embryonic dermal stimuli. Development 127: 5487-5495.

76. Hardy MH (1992) The secret life of the hair follicle. Trends in Genetics TIG 8: 55-61.
77. Potten CS (1974) The epidermal proliferative unit: the possible role of the central basal cell. Cell Tissue Kinet 7: 77-88.

78. Potten CS, Morris RJ (1988) Epithelial stem cells in vivo. J Cell Sci Suppl 10 45-62.

79. Schmidt GH, Blount MA, Ponder BA (1987) Immunochemical demonstration of the clonal organization of chimaeric mouse epidermis. Development 100 535-541.

80. Ghazizadeh S, Taichman LB (2001) Multiple classes of stem cells in cutaneous epithelium: a lineage analysis of adult mouse skin. EMBO J 20: 1215-1222.

81. Taylor G, Lehrer MS, Jensen PJ, Sun TT, Lavker RM (2000) Involvement of follicular stem cells in forming not only the follicle but also the epidermis. Cell 102: 451-461.

82. Lowry WE, Blanpain C, Nowak JA, Guasch G, Lewis L, et al. (2005) Defining the impact of beta-catenin/Tcf transactivation on epithelial stem cells. Genes Dev 19: 1596-1611.

83. Fre S, Huyghe M, Mourikis $P$, Robine $S$, Louvard D, et al. (2005) Notch signals control the fate of immature progenitor cells in the intestine. Nature 435: 964-968.

84. Andl T, Ahn K, Kairo A, Chu EY, Wine-Lee L, et al. (2004) Epithelial Bmpr1a regulates differentiation and proliferation in postnatal hair follicles and is essential for tooth development. Development 131: 2257-2268.

85. Nakamura S-i, Kawai T, Kamakura T, Ookura T (2009) TGF- $\beta 3$ is expressed in taste buds and inhibits proliferation of primary cultured taste epithelial cells. In Vitro Cellular \& Developmental Biology-Animal 46: 36-44.

86. Seta Y (2005) Regulation of Taste Bud Cell Differentiation by Notch Signaling Pathway. Chem Senses 30: i48-i49.

87. Schneider FT, Schänzer A, Czupalla CJ, Thom S, Engels K, et al. (2010) Sonic Hedgehog Acts as a Negative Regulator of $\beta$-Catenin Signaling in the Adult Tongue Epithelium. The American Journal of Pathology 177: 404-414.

88. Liu F, Thirumangalathu S, Gallant NM, Yang SH, Stoick-Cooper CL, et al (2007) Wnt-beta-catenin signaling initiates taste papilla development. Nat Genet 39: 106-112. 